\title{
LANDFILLS MULTIPLE GOAL OPTIMIZATION USING EQUATIONLESS QUALITATIVE RELATIONS
}

\author{
Mirko Dohnal \\ Center of Knowledge Economics, Faculty of Business and Management, \\ Brno University of Technology, Brno, Czech Republic
}

Received 2013-12-05; Revised 2014-01-26; Accepted 2014-02-11

\begin{abstract}
Landfills are unique and difficult to measure. Their optimization must be solved with a severe lack of information. The privilege of not utilizing information items based on common sense cannot be afforded, as this represents an important part of the available ad hoc landfill knowledge related to e.g., economics, sociology. Therefore, a flexible, formal tool for dealing with the common sense knowledge and data of a nonnumerical nature is required. The classical quantitative tools, e.g., statistics, are inefficient for dealing with such sets of non-quantitative information items as expert's interviews. Qualitative quantification is information non-intensive. It is based on just three values-positive, zero and negative; increasing, constant and decreasing. A qualitative model can be used to generate all possible qualitative activities/scenarios. It means that the past history and future scenarios of the landfill under study are known, given the model is correct. The scenarios can be screened against the prescribed trends (maximization or minimization) of objective functions, to identify all possible ways of achieving optimal results. A case study with four mutually competing objective functions is presented in details. No prior knowledge of qualitative reasoning is required.
\end{abstract}

Keywords: Multiple Goal, Landfill, Qualitative Model, Qualitative Scenario, Common Sense

\section{INTRODUCTION}

There are several levels of landfill studies. The highest level of generality is represented by the relations of landfills with a broad spectrum of parameters: Macroand microeconomics, sociology. The lowest level of generality and the most accurate model, is based exclusively on engineering measurements and knowledge, e.g., chemical engineering, chemistry (Chang et al., 2008; Wang et al., 2009). Engineering based models are by their nature very specific; however, any decision related to landfills must be based on the parameters of the particular location being studied (Wang et al., 2009).

Contaminated Sites Management (CSM) is inevitably a balancing act of many diverse factors such as social, economic, political, legal, technical and scientific issues, (Promentilla et al., 2008). This is the reason why CSM is closely related to various decision making algorithms and finally, to optimizations. Unfortunately subjective information items must be incorporated into studies to minimize sever information shortage. This requires application of different expert systems; Ooshaksaraie and Basri (2011).

Ecological related networks of knowledge items are inconsistent, fuzzy, uncertain, sparse, empirical and non-formalized. There is an urgent need for and efficient computer-based tools for making this knowledge explicit, well organized, processable, Salles and Bredeweg (2006).

There are many well-established methods of multi-objective optimization, Tabucanon (1989) and Jahn (2004). There are also numerous methods how to deal with complex systems, e.g., decompositions, Brusilovsky and Ostrovsky (1983). However, the results of the multiple goals optimization are as good as used mathematical models. Ill-known, Nonlinear and Multidimensional Systems (INMS) are, by virtue, difficult to measure/observe. However, even a wellrecognized system must be treated, rather often, as INMS during the early stages of project development and/or under severe time pressure Hong et al. (2008). 
The main reason for the special aspects of an INMS landfill model development is the general lack of knowledge. This tends to prevent the correct application of statistical methods. The information shortage can only be eliminated by additional sources. This means using additional measurements, or by utilizing the variables for which conventional methods cannot be applied. Qualitative models are such tools (Salles and Bredeweg, 2006).

\section{QUALITATIVE MODELS}

Qualitative models based on the following four quantifiers only, (Dohnal, 1991) Equation 1:

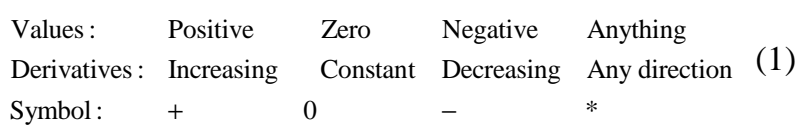

The following is a typical example of a qualitative knowledge item which can be expressed by a certain simple relationship between variables $\mathrm{X}$ and $\mathrm{Y}$ :

If the cost of leachate treatment $\mathrm{X}$ is increasing, then the water quality of $\mathrm{Y}$ also increases. However, the quality of the leachate cannot exceed a certain limit given by the current state of the arts.

It is possible to solve a set of differential and algebraic equations on a qualitative level, Dohnal (1991). However, this paper only looks at non-equation relations. These have proven to be quite significant in relation to landfills studies.

Extensive cooperation with landfill experts is required for the development of qualitative non-equation models. Systematic results and a minimal response waiting period have both proven to be advantages when using/applying these models, Bile et al. (2011).

All equationless relations are pair wise qualitative relations. It means that only the variables $\mathrm{X}$ and $\mathrm{Y}$ are involved, with no quantitative knowledge, Fig. 1. For example, the relation No. 22 indicates that Equation 2:

The relation is increasing

There is a linear relationship between $\mathrm{Y}$ and $\mathrm{X}$

If $\mathrm{X}=0$ then $\mathrm{Y}$ is positive

All three above given remarks are qualitative.

Figure $\mathbf{1}$ is a graphic representation of the 6 different shapes, namely shapes Nos. 21-26. The following matrix summarizes all possible shapes based on the first and second derivatives Equation 3:

$\begin{array}{llll}\text { Shape } & & \text { DY/DY1 } & \text { DDY/DDX } \\ \text { No. } & \text { Y if X }=0 & \text { Derivative } & 2^{\text {nd }} \text { Derivative } \\ 21 & + & + & + \\ 22 & + & + & 0 \\ 23 & + & + & - \\ 24 & + & - & + \\ 25 & + & - & 0 \\ 26 & + & - & - \\ 27 & 0 & + & + \\ 28 & 0 & + & 0 \\ 29 & 0 & + & - \\ 30 & 0 & - & + \\ 31 & 0 & - & 0 \\ 32 & 0 & - & - \\ 33 & - & + & + \\ 34 & - & + & 0 \\ 35 & - & + & - \\ 36 & - & - & + \\ 37 & - & - & 0 \\ 38 & - & - & -\end{array}$

The landfills information shortage is so severe that any available information must be taken into consideration. For example if it is known that Equation 4:

If $\mathrm{X}=0$ then $\mathrm{Y}=0$

in which case it is not the shape 22, Fig. 1, but the shape 28, see (3), that is used to best represent the corresponding relations.

A set of $m$ qualitative $n$-dimensional scenarios is described by the following set of triplets Equation 5:

$$
\begin{aligned}
& {\left[\left(\mathrm{X}_{1}, \mathrm{DX}_{1}, \mathrm{DDX}_{1}\right), \ldots\left(\mathrm{X}_{\mathrm{n}}, \mathrm{DX}_{\mathrm{n}}, \mathrm{DDX}_{\mathrm{n}}\right)\right]_{\mathrm{j}},} \\
& \mathrm{j}=1,2, \ldots, \mathrm{m}
\end{aligned}
$$

where $\mathrm{X}_{\mathrm{i}}$ is the $\mathrm{i}$-th variable and $\mathrm{DX}_{\mathrm{i}}$ and $\mathrm{DDX}_{\mathrm{i}}$ are the first qualitative and second qualitative derivations of $\mathrm{X}_{\mathrm{i}}$ with respect to time $(t)$. Higher derivatives are irrelevant in this case. They are usually unknown if the INMS is studied. Landfills, studied on the highest level of generality, are INMS.

The relations given in Fig. 1 are between two variables. However, the triples used to characterize a scenario (5) are time functions. For example, the triplet $\mathrm{s}$ $(+++)$ characterize the same shape as the shape 21, Fig. $\mathbf{1}$, if the variable $\mathrm{X}$ is time.

The given shapes (3) do not cover e.g., minimum/qualitative valley or maximum/qualitative hill. 

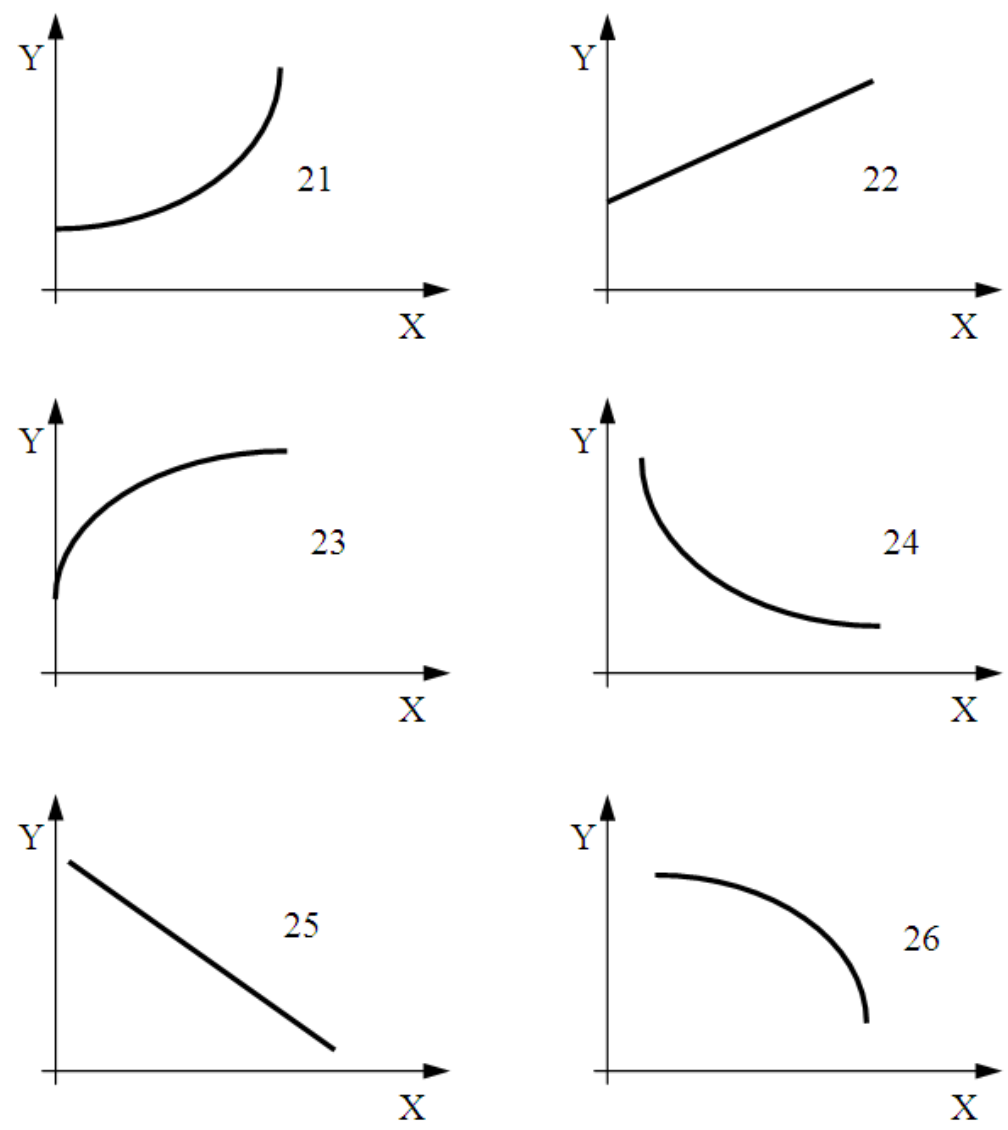

Fig. 1. Examples of pair wise qualitative relations

However, a sequence of the shape 23 followed by the shape 26 represents a qualitative maximum.

Set Equation 6:

$$
\mathrm{S}(\mathrm{m}, \mathrm{n})
$$

is a set of $m$ qualitative $n$-dimensional scenario

Let the set $S(6)$ be a solution of a qualitative $n$ dimensional model M Equation 7:

$$
\mathrm{M}(\mathrm{r}, \mathrm{n})
$$

where, $\mathrm{r}$ is the number of its non-equation relations.

There are $3 \times 3 \times 3=27$ different triplets $(X, D X$, DDX), as each element of the triplet can have three values (1). There are $27^{\mathrm{n}}$ different $\mathrm{n}$ dimensional scenarios. However, a model (7) usually rejects the majority of such n-dimensional triplets. Let us suppose that the $\mathrm{m}$ n-dimensional triplets have not been rejected by the model (6). Such n-dimensional triplets are the scenarios $\mathrm{S}(6)$

It is not the goal of this paper to study the algorithm of how to solve qualitative models, i.e., how model scenarios are rejected. It is a combinatorial problem. The most trivial algorithm is based on the systematic confrontation of all possible n-dimensional triplets and the model itself. This type of solution is called brutal force in artificial intelligence Dohnal (1988) and Parsons (1995).

\section{QUALITATIVE TRANSITIONS}

The unsteady state behavior of an INMS can be described by a time sequence of scenarios. A transitional graph gives all the possible unsteady state behaviors. If each scenario is represented by a node and all transitions are graphically represented by oriented arks between corresponding pairs of scenarios, the result is an oriented graph of all possible transitions. 
Mirko Dohnal / American Journal of Environmental Science 10 (1): 26-34, 2014

Table 1. A list of all one dimensional transitions

\begin{tabular}{|c|c|c|c|c|c|c|c|c|}
\hline No. & $\begin{array}{l}\text { From } \\
\text { (a) }\end{array}$ & $\begin{array}{l}\text { To } \\
\text { (b) }\end{array}$ & $\begin{array}{l}\text { Or } \\
\text { (c) }\end{array}$ & $\begin{array}{l}\text { Or } \\
\text { (d) }\end{array}$ & $\begin{array}{l}\text { Or } \\
\text { (e) }\end{array}$ & $\begin{array}{l}\text { Or } \\
\text { (f) }\end{array}$ & $\begin{array}{l}\text { Or } \\
(\mathrm{g})\end{array}$ & $\begin{array}{l}\text { Or } \\
\text { (h) }\end{array}$ \\
\hline 1 & +++ & ++0 & & & & & & \\
\hline 2 & ++0 & +++ & ++- & & & & & \\
\hline 3 & ++- & ++0 & $+0-$ & +00 & & & & \\
\hline 4 & $+0+$ & +++ & & & & & & \\
\hline 5 & +00 & +++ & +- & & & & & \\
\hline 6 & $+0-$ & +- & & & & & & \\
\hline 7 & +-+ & +-0 & $+0+$ & +00 & $0-+$ & $00+$ & 000 & $0-0$ \\
\hline 8 & +-0 & +-+ & +- & $0-0$ & & & & \\
\hline 9 & +- & +-0 & $0--$ & $0-0$ & & & & \\
\hline 10 & $0++$ & ++0 & ++- & +++ & & & & \\
\hline 11 & $0+0$ & ++0 & ++ & +++ & & & & \\
\hline 12 & $0+-$ & ++- & & & & & & \\
\hline 13 & $00+$ & +++ & & & & & & \\
\hline 14 & 000 & +++ & - . - & & & & & \\
\hline 15 & $00-$ & _. . & & & & & & \\
\hline 16 & $0-+$ & --+ & & & & & & \\
\hline 17 & $0-0$ & --0 & --+ & - . - & & & & \\
\hline 18 & 0 - - & --0 & --+ & - . - & & & & \\
\hline 19 & -++ & -+0 & $0++$ & $0+0$ & & & & \\
\hline 20 & -+0 & -+- & -++ & $0+0$ & & & & \\
\hline 21 & -+- & -+0 & -0 - & -00 & $0+-$ & $00-$ & 000 & $0+0$ \\
\hline 22 & $-0+$ & -++ & & & & & & \\
\hline 23 & -00 & -++ & $\ldots$ & & & & & \\
\hline 24 & $-0-$ & $\ldots$ & & & & & & \\
\hline 25 & --+ & --0 & $-0+$ & -00 & & & & \\
\hline 26 & --0 & -- & --+ & & & & & \\
\hline 27 & $\ldots$ & --0 & & & & & & \\
\hline
\end{tabular}

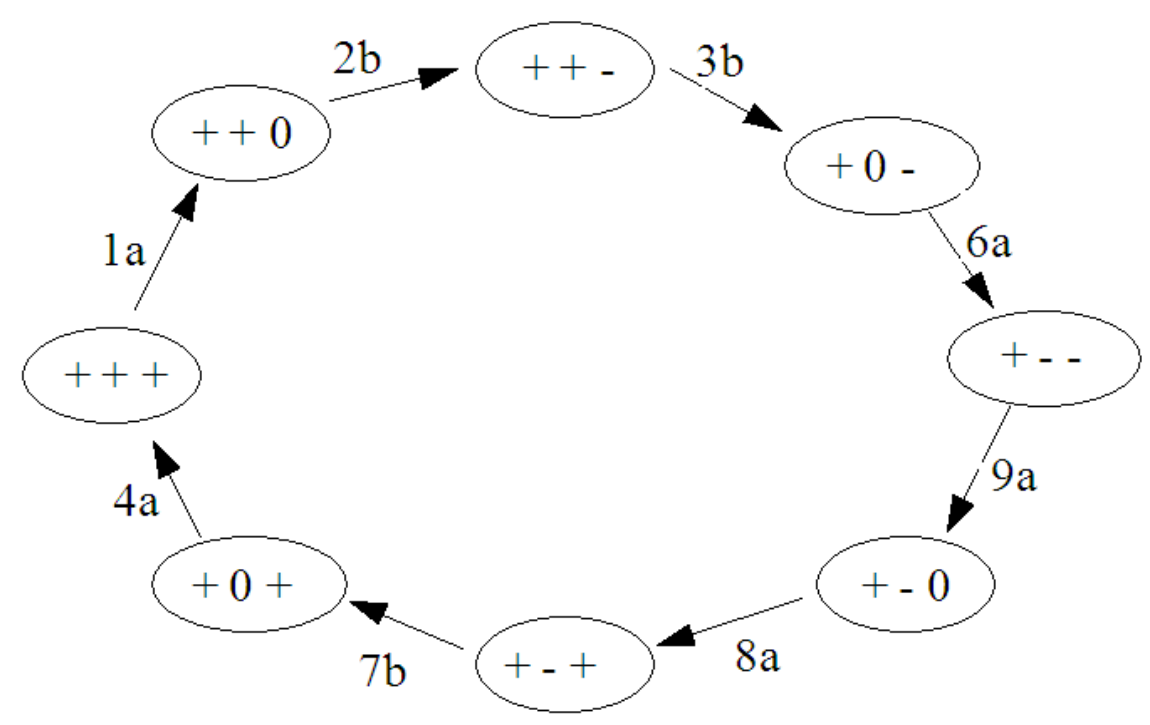

Fig. 2. Qualitative one dimensional time record of an oscillation

Any time behavior of the INMS can be characterized as a path in the transition graph. The transition graph is thus a condensed description of all possible unsteady state behaviors. 
A complete set of all possible one dimensional transitions is given in the following table.

The third line of Table $\mathbf{1}$ indicates that it is possible to transfer the triplet $(++-)$ into the triplet $(+0-)$. This transition is not the only possibility. There are two other possible transitions:

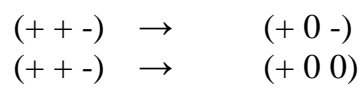

Figure 2 gives a qualitative description of an oscillation using the one dimensional triplets $n=1$ (5).

Table 1 is not a dogma. It could be modified on an ad hoc basis. The only requirement is that the transitions must satisfy the common sense reasoning of a user.

A transitional graph $\mathrm{G}$ is an oriented graph. Its nodes are the set of scenarios $\mathrm{S}$ and oriented arcs are the transitions T Equation 8:

$\mathrm{G}(\mathrm{S}, \mathrm{T})$

However, the set of transitions $\mathrm{T}$, can be easily generated by the corresponding set of scenarios $\mathrm{S}$ using Table 1.

Transition numbers shown in Fig. 2 correspond to transitions in Table 1. For example transition $3 \mathrm{~b}$ is the row 3 , column b.

\section{QUALITATIVE OPTIMIZATION}

The following demonstration is based on a simple set of equationless relations in a model $\mathrm{M}(2,3)$; (7) Equation 9:

$$
\begin{array}{lll}
\text { Shape No. } & X & Y \\
22 \text { (see Fig. 1) } & X_{1} & X_{2} \\
26 \text { (see Fig. 1) } & X_{3} & X_{2}
\end{array}
$$

To simplify the problem, let us suppose that all three variables $X_{1}, X_{2}$ and $X_{3}$ are positive. For example, $X_{1}$ is a management qualification and this is always positive. Therefore, the following triplets are taken into consideration $\left(+, \mathrm{DX}_{1}, \mathrm{DDX}_{1}\right)$.

The model (9) has 13 scenarios $S(13,3) ;(6))$ as its solution Equation 10:

$\begin{array}{llll} & \mathrm{X}_{1} & \mathrm{X}_{2} & \mathrm{X}_{3} \\ 1 & +++ & +++ & +- \\ 2 & ++0 & ++0 & +- \\ 3 & ++- & ++- & +-+ \\ 4 & ++- & ++- & +-0 \\ 5 & ++- & ++- & +- \\ 6 & +0+ & +0+ & +0- \\ 7 & +00 & +00 & +00 \\ 8 & +0- & +0- & +0+ \\ 9 & +-+ & +-+ & ++- \\ 10 & +-0 & +-0 & ++- \\ 11 & +-- & +-- & +++ \\ 12 & +-- & +-- & ++0 \\ 13 & +-- & +- & ++-\end{array}$

The set of three dimensional scenarios (10) generates the following transitional graph (8):

Let the third variable $\mathrm{X}_{3}(10)$ be an objective function, which must be maximized using the variables $X_{1}$ and $X_{2}$.

$\operatorname{Max} X_{3}$

$f\left(X_{1}, X_{2}, X_{3}\right)=0$

Using the well-established optimization terminology, Jahn (2004), where the variable $X_{3}$ is an objective function, $X_{1}$ and $X_{2}$ are independent variables and $f$ represents the set of constraints.

The first scenario is least desirable (10). The objective function $\mathrm{X}_{3}$ is decreasing more and more rapidly (+ - -). The scenario $11(10)$ is the best possibility, as it increases the objective function more and more rapidly $\mathrm{DX}_{3}=+, \mathrm{DDX}_{3}=+$. Let us suppose that the current situation is characterized by the first scenario and the goal is to reach the scenario No. 11 by changing the independent variables $X_{1}$ and $X_{3}$.

Figure 3 indicates that there are several paths how to reach node 11 from node 1 . Two short paths are:

$1 \rightarrow 2 \rightarrow 4 \rightarrow 3 \rightarrow 7 \rightarrow 11$

$1 \rightarrow 2 \rightarrow 4 \rightarrow 3 \rightarrow 8 \rightarrow 11$

The following time sequence of scenarios is the detailed description of the first path:

$\begin{array}{llll} & \mathrm{X}_{1} & \mathrm{X}_{2} & \mathrm{X}_{3} \\ 1 & +++ & +++ & +-- \\ 2 & ++0 & ++0 & +-- \\ 4 & ++- & ++- & +-0 \\ 3 & ++- & ++- & +-+ \\ 7 & +00 & +00 & +00 \\ 11 & +- & +-- & +++\end{array}$




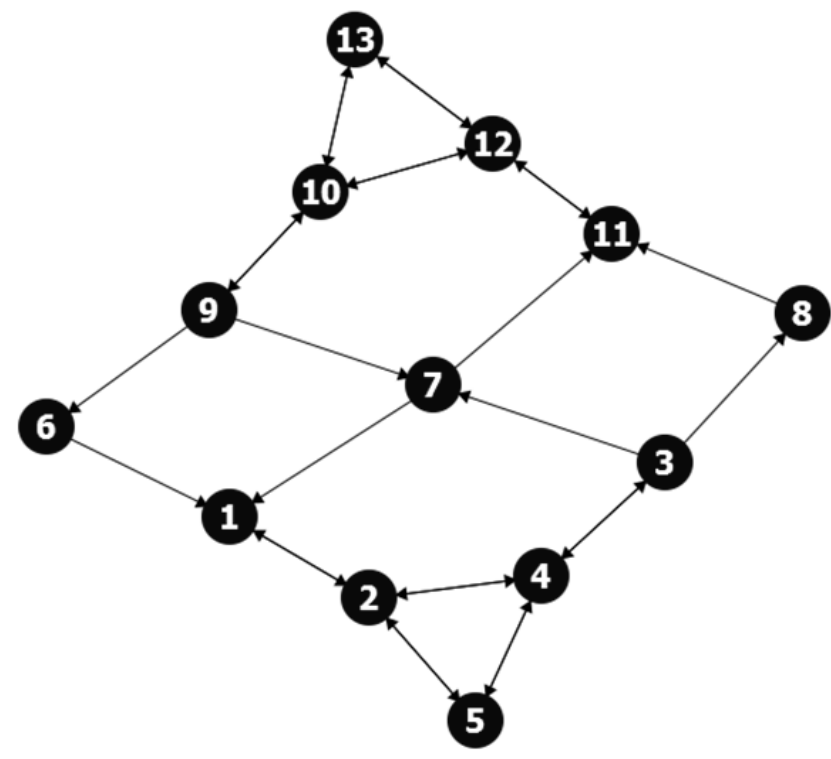

Fig. 3. The graph based on the set of scenarios (10)

If both variables $X_{1}, X_{2}$ are under control of a decision maker, then it would be easy to move from the first scenario to scenario No. 11. However, if e.g., variable $X_{2}$ is not controlled by a decision maker then a much more complex problem must be studied. However, even this problem is fully described by the graph in Fig. 3.

\section{QUALITATIVE MULTIPLE GOAL OPTIMIZATION}

A trivial model of a landfill is studied. Let us suppose that there are two independent variables $\mathrm{X}_{1}, \mathrm{X}_{2}$ and two objective functions Q1, Q2. For example:

$\mathrm{Q}_{1}$ Quality of Life

$\mathrm{Q}_{2}$ Quality of Leachate

$\mathrm{X}_{1}$ Feasibility of the relevant Membrane Technologies

$\mathrm{X}_{2}$ Investment Cost

Because of their very nature, both objective functions must be maximized Equation 11:

$\operatorname{Max} \mathrm{Q}_{1}$

$\operatorname{Max} \mathrm{Q}_{2}$

The vector $\mathrm{F}$ of constraints is represented by a set of equationless relations, Fig. 1 Equation 12:

$$
\mathrm{F}\left(\mathrm{X}_{1}, \mathrm{X}_{2}, \mathrm{Q}_{1}, \mathrm{Q}_{2}\right)=0
$$

Let us suppose that the model (12) has the following scenarios as its solution Equation 13:

$$
\begin{array}{lllll} 
& \mathrm{X}_{1} & \mathrm{X}_{2} & \mathrm{Q}_{1} & \mathrm{Q}_{2} \\
1 & +++ & +++ & +-- & +-- \\
2 & ++- & +-- & +++ & +-- \\
3 & +-- & +-+ & +-+ & +++
\end{array}
$$

The first qualitative solution is totally unacceptable, see (11). Both objective functions decrease if independent variables $\mathrm{X}_{1}$ and $\mathrm{X}_{2}$ follow the qualitative time pattern given in (13). Therefore, the qualitative behavior of independent variables $X_{1}$ and $\mathrm{X}_{2}$ Equation 14:

$$
\begin{array}{lll} 
& \mathrm{X}_{1} & \mathrm{X}_{2} \\
1 & +++ & +++
\end{array}
$$

is bad with respect to the maximization of two objective functions $\mathrm{Q}_{1}$ and $\mathrm{Q}_{2}$.

The best possible scenario is Equation 15:

$$
\begin{array}{llll}
\mathrm{X}_{1} & \mathrm{X}_{2} & \mathrm{Q}_{1} & \mathrm{Q}_{2} \\
++- & +-0 & +++ & +++
\end{array}
$$

This scenario maximizes both objective functions in the best possible way, since all the derivatives are 
positive. However, such scenario does not exist, see (13). The third scenario (13) is a compromise, the second objective function is maximized, but the first objective function minimized.

\section{RESULTS}

It is obvious that a set of qualitative models is quite broad. There are several specific ecology related topics studied by qualitative models, Salles and Bredeweg (2006) and Bile et al. (2011). Different models are based on different sets of variables $\mathrm{Y}$ and different points of view/objective functions Q; Dokas et al. (2009); Chen et al. (2008) and Hokkanen and Salminen (1997):

Water Reuse, Groundwater Resources, Irrigation, Biodiversity, Non Invasive In Situ Technologies, Low-energy Technologies, Preserve Natural Resources, Renewable Energy, Emissions of Greenhouse Gases, Offsite Migration of Contamination, Employment and Educational Opportunities Health and Safety Risks During Remedy Implementation, Site Available for Reuse, Number of Sites Available for Reuse, Locally Sourced Materials, Noise, Odor, Public Access to Open Space, Goodwill

However, such a rich set of variables requires a description of complex models and consequently a rich set of scenarios.

The following simple and easy to understand set of variables are used in this study Equation 16:

\author{
$\mathrm{Y}_{1}$ Waste volume \\ $\mathrm{Y}_{2}$ Transportation of solid waste \\ $\mathrm{Y}_{3}$ Waste combustion \\ $\mathrm{Q}_{1} \quad$ Air quality \\ $\mathrm{Q}_{2}$ Water quality \\ $\mathrm{Q}_{3}$ Quality of life \\ $\mathrm{Q}_{4}$ Employment \\ $\mathrm{Q}_{5} \quad$ Water availability
}

Let us suppose that $\mathrm{Y}$ is the set of independent variables and $\mathrm{Q}$ is the set of objective functions.

A simple qualitative model M $(10,8)$, see (7) Fig. 1 Equation 17:

$\begin{array}{llll}1 & 22 & Y_{1} & Y_{2} \\ 2 & 23 & Y_{1} & Y_{3} \\ 3 & 24 & Y_{1} & Y_{4} \\ 4 & 24 & Y_{1} & Y_{4}\end{array}$

$\begin{array}{llll}5 & 24 & \mathrm{Y}_{1} & \mathrm{Q}_{1} \\ 6 & 23 & \mathrm{Y}_{1} & \mathrm{Q}_{4} \\ 7 & 23 & \mathrm{Q}_{2} & \mathrm{Q}_{5} \\ 8 & 21 & \mathrm{Q}_{2} & \mathrm{Q}_{3} \\ 9 & 21 & \mathrm{Q}_{1} & \mathrm{Q}_{3} \\ 10 & 22 & \mathrm{Q}_{1} & \mathrm{Q}_{2}\end{array}$

indicates that 10 equationless relations are used to formalize relations among the set of variables (16). The set of 97 scenarios is generated using software described in (Vicha and Dohnal, 2008). Only the first 6 scenarios out of 97 are given Equation 18:

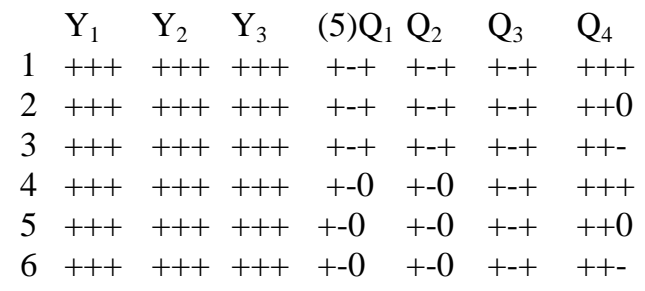

The first three scenarios clearly indicate that the model (17) does not restrict the second derivative of the fourth objective function $\mathrm{Q}_{4}$. The only difference among the first three scenarios (20), is the change of the $Q_{4}$ second derivatives. This second derivative of $\mathrm{Q}_{4}$ has all possible values, see (1). However, this is possible only if all three independent variables (Y) follow the triplet $(++$ $+)$. Such results can be used to develop the qualitative model (17) by adding such equationless relations, which eliminate free change of the second derivative of $\mathrm{Q}_{4}$.

The very nature of all five objective functions (Q) requires maximization, see (16) for explanation. However, there is no such scenario in the set (18), which has a positive derivative for all five objective functions.

The first scenario (18) maximizes just one objective function namely $\mathrm{Q}_{4}$ :

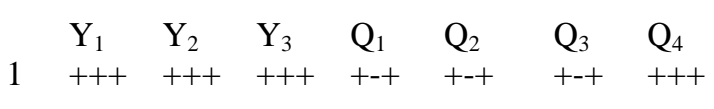

The scenario 51, maximizes three goal functions namely $\mathrm{Q}_{1}, \mathrm{Q}_{2}$ and $\mathrm{Q}_{3}$. The objective function $\mathrm{Q}_{4}$ is decreasing, however, its' decrease is gradually slowing down as its second derivative is negative:

$$
\begin{array}{llllllll} 
& \mathrm{Y}_{1} & \mathrm{Y}_{2} & \mathrm{Y}_{3} & \mathrm{Q}_{1} & \mathrm{Q}_{2} & \mathrm{Q}_{3} & \mathrm{Q}_{4} \\
51 & +-+ & +-+ & +-+ & +++ & +++ & +++ & +-+
\end{array}
$$

However, the time behaviors of the first, second and the third scenarios are increasing more and more rapidly, as their triplets are: $(+++)$. 
The scenarios 52 and 53 are not so good in terms of the fourth objective function, see below:

$\begin{array}{llllllll} & \mathrm{Y}_{1} & \mathrm{Y}_{2} & \mathrm{Y}_{3} & \mathrm{Q}_{1} & \mathrm{Q}_{2} & \mathrm{Q}_{3} & \mathrm{Q}_{4} \\ 52 & +-+ & +-+ & +-+ & +++ & +++ & +++ & +-0 \\ 53 & +-+ & +-+ & +-+ & +++ & +++ & +++ & +--\end{array}$

For example, the time behavior of the fourth scenario is characterized by the linear decrease $(+-0)$.

\section{CONCLUSION}

If trends are not known then nothing is known and it is not possible to make any predictions and/or decisions which are not totally subjective. Qualitative models are based on trends. It means that qualitative models are the least information intensive models. Qualitative models require very modest information input.

The main advantages of the landfill qualitative modeling are:

- No numerical values of constants and parameters are needed

- The set of solutions/scenarios is provably complete, i.e., there cannot be any other qualitative scenarios not generated by the qualitative model. No scenario can be overlooked if predictions/decision/optimization is based on qualitative models

- It is relatively easy to develop the first version of a multidimensional qualitative model of interdisciplinary nature. The model is a set of qualitative equationless relations and their mutual (in) consistency is checked by qualitative reasoning algorithms. Therefore a very useful feed-back from a computer to a model developer is available

- Qualitative models can be used as idea generators for:

- Development of quantitative models, e.g. the best possible generator of specific differential equations for identification of numerical constants using traditional statistical methods

- Elimination of variables which have no any influence on extremalisation (minimization/maximization) of an objective function under study.

Qualitative models represent just one formal tool. A broad spectrum of research on artificial intelligence has generated many different methods, algorithms and methodologies that can be used successfully to integrate different landfills knowledge itesm of different origins including such non engineering extremely vague aspects as sociology.

\section{REFERENCES}

Bile, J., J. Jura, J. Pokorny and I. Bukovsky, 2011. Qualitative modeling and monitoring of selected ecosystem functions. Ecol. Model., 222: 3640-3650. DOI: 10.1016/j.ecolmodel.2011.08.002

Brusilovsky, A. M. and G. M. Ostrovsky, 1983. The decomposition optimization of complex systems. Int. J. Syst. Sci., 14: 1273-1286. DOI: 10.1080/00207728308926529

Chang, N.B., G. Parvathinathan and J.B. Breeden, 2008. Combining GIS with fuzzy multicriteria decisionmaking for landfill siting in a fast-growing urban region. J. Environ. Manage., 87: 139-153. DOI: 10.1016/j.jenvman.2007.01.011

Chen, S.H., A.J. Jakeman and J.P. Norton, 2008. Artificial Intelligence techniques: An introduction to their use for modelling environmental systems. Math. Comput. Simulat., 78: 379-400. DOI: 10.1016/j.matcom.2008.01.028

Dohnal, M., 1988. Naive models as active expert system in bioengineering and chemical engineering. Coll. Czech. Chem. Comm., 53: 1476-1499. DOI: $10.1135 /$ cccc19881476

Dohnal, M., 1991. A methodology for common-sense model development. Comput. Indus., 16: 141-158. DOI: 10.1016/0166-3615(91)90086-O

Dokas, I.M., D.A. Karras and D.C. Panagiotakopoulos, 2009. Fault tree analysis and fuzzy expert systems: Early warning and emergency response of landfill operations. Environ. Modell. Softw., 24: 8-25. DOI: 10.1016/j.envsoft.2008.04.011

Hokkanen, J. and P. Salminen, 1997. Choosing a solid waste management system using multicriteria decision analysis. Eur. J. Operat. Res., 98: 19-36. DOI: 10.1016/0377-2217(95)00325-8

Hong, X., R.J. Mitchell, S. Chen, C.J. Harris and K. Li et al., 2008. Model selection approaches for non-linear system identification: A review. Int. J. Syst. Sci., 39: 925-946. DOI: 10.1080/00207720802083018

Jahn, J., 2004. Vector Optimization: Theory, Applications and Extensions. 1st Edn., Springer, Berlin, ISBN-10: 3540206159, pp: 465.

Ooshaksaraie, L. and N.E.A. Basri, 2011. An expert system applied in construction water quality monitoring. Am. J. Environ. Sci., 7: 75-81. DOI: 10.3844/ajessp.2011.75.81 
Parsons, S., 1995. The qualitative and semiqualitative analysis of environmental problems. Environ. Softw., 10: 75-85. DOI: 10.1016/02669838(95)00008-9

Promentilla, M.A.B., T. Furuichi, K. Ishii and N. Tanikawa, 2008. A fuzzy analytic network process for multi-criteria evaluation of contaminated site remedial countermeasures. J. Environ. Manage., 88: 479-495. DOI: 10.1016/j.jenvman.2007.03.013

Salles, P. and B. Bredeweg, 2006. Modelling population and community dynamics with qualitative reasoning. Ecol. Model., 195: 114-128. DOI: 10.1016/j.ecolmodel.2005.11.014
Tabucanon, M.T., 1989. Multiple Criteria Decision Making in Industry. 1st Edn., Elsevier, Amsterdam, ISBN-10: 0444705414, pp: 354.

Vicha, T. and M. Dohnal, 2008. Qualitative feature extractions of chaotic systems. Chaos, Soliton. Fract., 38: 364-373. DOI: 10.1016/j.chaos.2008.01.008

Wang, G., L. Qin, G. Li and L. Chen, 2009. Landfill site selection using spatial information technologies and AHP: A case study in Beijing, China. J. Environ. Manage., 90: 2414-2421. DOI: 10.1016/j.jenvman.2008.12.008 